\title{
Hemodynamic and morphological characteristics of unruptured posterior communicating artery aneurysms with oculomotor nerve palsy
}

\author{
Nan Lv, MD, ${ }^{1}$ Ying Yu, MD, ${ }^{1}$ Jinyu Xu, MD, ${ }^{1}$ Christof Karmonik, PhD, ${ }^{2}$ Jianmin Liu, MD, ${ }^{1}$ and \\ Qinghai Huang, MD'
}

${ }^{1}$ Department of Neurosurgery, Changhai Hospital, Second Military Medical University, Shanghai, China; and ${ }^{2}$ Cerebrovascular
Center, Department of Neurosurgery, Houston Methodist, Houston, Texas

OBJECTIVE Unruptured posterior communicating artery (PCOA) aneurysms with oculomotor nerve palsy (ONP) have a very high risk of rupture. This study investigated the hemodynamic and morphological characteristics of intracranial aneurysms with high rupture risk by analyzing PCoA aneurysms with ONP.

METHODS Fourteen unruptured PCoA aneurysms with ONP, 33 ruptured PCoA aneurysms, and 21 asymptomatic unruptured PCoA aneurysms were included in this study. The clinical, morphological, and hemodynamic characteristics were compared among the different groups.

RESULTS The clinical characteristics did not differ among the 3 groups $(p>0.05)$, whereas the morphological and hemodynamic analyses showed that size, aspect ratio, size ratio, undulation index, nonsphericity index, ellipticity index, normalized wall shear stress (WSS), and percentage of low WSS area differed significantly $(p<0.05)$ among the 3 groups. Furthermore, multiple comparisons revealed that these parameters differed significantly between the ONP group and the asymptomatic unruptured group and between the ruptured group and the asymptomatic unruptured group, except for size, which differed significantly only between the ONP group and the asymptomatic unruptured group $(p=$ 0.0005). No morphological or hemodynamic parameters differed between the ONP group and the ruptured group.

CONCLUSIONS Unruptured PCoA aneurysms with ONP demonstrated a distinctive morphological-hemodynamic pattern that was significantly different compared with asymptomatic unruptured PCoA aneurysms and was similar to ruptured PCOA aneurysms. The larger size, more irregular shape, and lower WSS might be related to the high rupture risk of PCoA aneurysms.

http://thejns.org/doi/abs/10.3171/2015.6.JNS15267

KEY WORDS intracranial aneurysm; rupture; morphology; hemodynamics; computational fluid dynamics; vascular disorders

$\mathrm{O}$ CULOMOTOR nerve palsy (ONP) is a presenting sign of posterior communicating artery ( $\mathrm{PCoA})$ aneurysms, which have a high risk of rupture. ${ }^{10,16}$ An understanding of the characteristics of PCoA aneurysms with ONP may be helpful to identify the features of aneurysms prone to rupture and to provide meaningful information for predicting rupture, even in intracranial aneurysms (IAs) at other locations. The morphology and hemodynamics of IAs are currently being actively investigated with respect to rupture. ${ }^{18,20}$ Quantitative parameters that can accurately assess rupture risk are being determined. In this study, we examined hemodynamic and morphological features of unruptured PCoA aneurysms with ONP by comparing them with asymptomatic unruptured PCoA aneurysms and ruptured PCoA aneurysms.

\section{Methods}

The institutional review board of Changhai Hospital, which is affiliated with the Second Military Medical University, approved this study. The requirement for patient informed consent was waived. 


\section{Patients and Imaging}

From January 2009 to December 2012, 14 patients in Changhai Hospital who suffered from sudden ONP were diagnosed with unruptured PCoA aneurysms by 3D rotational angiography (RA). The mean interval from the onset of ONP to the performance of 3D RA was 12.7 days. For further comparison of morphological and hemodynamic features, we retrospectively analyzed a cohort of consecutive patients with PCoA aneurysms who were treated between January 2011 and December 2012 in our hospital; their rupture statuses were determined according to the radiographic findings. Ruptured PCoA aneurysms with ONP were excluded because the ONP in patients with subarachnoid hemorrhage could result from inflammatory and microvascular effects that are secondary to the blood in the cisterns. ${ }^{5}$ In addition, only patients with complete medical records and 3D RA data were included.

The 3D RA was performed with the Artis zee biplane angiographic system (VC14, Siemens). A 5-second digital subtraction angiography acquisition protocol was adopted, and $18 \mathrm{ml}$ of contrast agent was injected through the internal carotid artery at a rate of $3 \mathrm{ml} / \mathrm{sec}$. During the 5-second acquisition, after a delay of 1 second, a $200^{\circ}$ rotation of the $\mathrm{C}$-arm was performed to obtain 133 frames. All of the acquired 5-second digital subtraction angiography data were transferred to a syngo X Workplace (VB15, Siemens) for reconstruction of the 3D internal carotid arterial tree and exported in a stereolithography format for computational fluid dynamics (CFD) simulations.

\section{Modeling of PCoA Aneurysms}

The 3D models were segmented and smoothed with Geomagic Studio version 9.0 (3D Systems, Inc.) and imported into ICEM CFD 11.0 (ANSYS, Inc.) to create volume grids for the fluid dynamics simulations. The total number of elements in each model was between 1,000,000 and $1,400,000$. The vessel wall was divided into 3 parts: the aneurysm, parent artery, and other vessels, as previously described. ${ }^{19}$

A pulsatile velocity waveform was obtained with transcranial Doppler imaging of a healthy subject. We then captured the flow spectrum envelope to obtain an average waveform of blood flow velocity in a complete cardiac cycle using MATLAB 7.0 software (The MathWorks, Inc.).

CFD simulations were performed using CFX 11.0 (ANSYS, Inc.). The vessel was considered a rigid wall with noslip boundary conditions. The governing equations underlying the calculation were the Navier-Stokes formulations with the assumption of laminar and incompressible blood flow. ${ }^{15}$ The density and dynamic viscosity were specified as $\rho=1050 \mathrm{~kg} / \mathrm{m}^{3}$ and $\mu=0.00345 \mathrm{~Pa} \cdot \mathrm{sec}$, respectively. The inlet was imposed by the pulsatile velocity profile that was obtained with transcranial Doppler and the outlet was defined as the opening boundary condition with 0 static pressure. We discretized the entire 0.8 -second cardiac cycle with a time step of 0.001 second for the numeric simulation. For each model, we simulated 3 continuous cardiac cycles to ensure the numeric stability of the simulation, and the last cycle was considered the output. The results were postprocessed and visualized using CFX 11.0.

\section{Hemodynamic Parameter Calculations}

Three hemodynamic parameters were calculated in this study: normalized wall shear stress (WSS), percentage of low WSS area (LSA), and oscillatory shear index (OSI). The time-averaged WSS was further averaged over the dome area (the entire luminal surface of the aneurysm sac) and then normalized by the average parent-vessel WSS in the same patient to allow comparisons among different patients. ${ }^{8,17}$ The LSA, which was defined as the areas of the aneurysm wall exposed to a WSS less than $10 \%$ of the mean parent-vessel WSS, was then normalized by the dome area. ${ }^{8,17}$ OSI, which is a nondimensional parameter, measures the directional change of WSS during the cardiac cycle ${ }^{6}$ :

$$
W S S=\frac{1}{T} \int_{0}^{T}\left|w S s_{i}\right| d t
$$

and

$$
O S I=\frac{1}{2}\left\{1-\frac{\left|\int_{0}^{T} w s s_{i} d t\right|}{\int_{0}^{T}\left|w s s_{i}\right| d t}\right\}
$$

where wss $_{\mathrm{i}}$ is the instantaneous WSS vector, $\mathrm{t}$ is time, and $\mathrm{T}$ is the duration of the cycle. The OSI was averaged over the dome area.

\section{Morphological Parameter Calculations}

Seven morphological parameters described by Dhar et $\mathrm{al}^{3}{ }^{3}$ were included in the study. In addition to common parameters, such as the size of the aneurysm, aspect ratio (AR), and size ratio (SR), we calculated the undulation index (UI), ellipticity index (EI), and nonsphericity index (NSI) based on 3D variables, such as the surface area and volume. These parameters quantitatively reflected the undulation, elongation of the aneurysmal sac, and presence of blebs. The neck plane was defined as the location where the aneurysmal sac pouched outward from the parent vessel. After defining the neck plane, the models were cut into the aneurysm and the inlet and outlet planes of the parent artery, and then exported in stereolithography formats. These formats were imported into MATLAB 7.0, which was used to calculate and visualize the 7 morphological parameters.

\section{Statistical Analysis}

The statistical analyses were performed using Microsoft Excel 2003 and SAS version 9.1 (SAS Institute, Inc.). The parameters were expressed as the median (interquartile range) or number of patients (\%), as appropriate. Count data were analyzed using chi-square tests. For the measurement data, Kruskal-Wallis $\mathrm{H}$ tests were used, followed by Nemenyi tests for multiple comparisons. A p value less than 0.05 (2-sided) was considered statistically significant. 


\section{Results}

In addition to the 14 unruptured PCoA aneurysms with ONP, 54 PCoA aneurysms without ONP in 51 patients were included in the study. Thirty-three were ruptured PCoA aneurysms (the ruptured group), and 21 were unruptured PCoA aneurysms (the asymptomatic unruptured group). Of the total 65 patients, 14 were men and 51 were women. The patients' ages ranged from 25 to 86 years, with a median age of 62 years.

None of the baseline characteristics (such as age, sex, hypertension, diabetes mellitus, or current smoking status) differed among the 3 groups. The morphological and hemodynamic analysis showed that size, AR, SR, UI, NSI, EI, normalized WSS, and LSA differed significantly among the 3 groups, whereas OSI did not differ significantly $(\mathrm{p}=0.050)($ Table 1$)$.

Further multiple comparisons were performed to explore the exact relationship between each group, as shown in Table 2. Between the ONP and ruptured group, none of the previously significant parameters differed significantly. This indicated that the ONP group had a similar morphological-hemodynamic pattern to the ruptured group. Between the ONP and asymptomatic unruptured group, all of the morphological parameters in the ONP group were significantly larger than those in the asymptomatic unruptured group. This indicated that PCoA aneurysms with ONP were larger and more irregularly shaped, which may have been caused by enlargement of the aneurysm sac or by the presence of blebs. Meanwhile, a low-WSS hemodynamic status (lower normalized WSS and higher LSA) was observed in the ONP group compared with the asymptomatic unruptured group. Finally, the morphological parameters in the ruptured group were significantly greater than those in the asymptomatic unruptured group, except for size $(p=0.050)$. A low-WSS hemodynamic sta-
TABLE 2. Results ( $p$ values) of multiple comparisons among the 3 groups of PCOA aneurysms

\begin{tabular}{lccc}
\hline \multicolumn{3}{c}{$p$ Value } \\
\hline \multicolumn{1}{c}{ Variable } & ONP vs Rup & ONP vs Unrup & Rup vs Unrup \\
\hline Size, $\mathrm{mm}$ & 0.109 & 0.0005 & 0.050 \\
\hline $\mathrm{AR}$ & 0.235 & 0.0001 & 0.004 \\
\hline $\mathrm{SR}$ & 0.993 & 0.028 & 0.007 \\
\hline $\mathrm{UI}$ & 0.309 & 0.0004 & 0.007 \\
\hline $\mathrm{NSI}$ & 0.191 & $<0.0001$ & 0.0005 \\
\hline EI & 0.399 & $<0.0001$ & $<0.0001$ \\
\hline Normalized WSS & 0.446 & 0.002 & 0.018 \\
\hline Percentage of LSA & 0.470 & 0.001 & 0.008 \\
\hline
\end{tabular}

tus was observed in the ruptured group compared with the asymptomatic unruptured group. Two exemplary cases from each group were plotted in terms of WSS (Fig. 1).

\section{Discussion}

Posterior communicating artery aneurysms with ONP have a relatively higher rupture risk compared with asymptomatic unruptured ones. ${ }^{10,16}$ We sought to understand the characteristics of high-rupture-risk aneurysms by comparing them with those that were asymptomatic. Most hemodynamic studies on rupture risk have compared postruptured and unruptured IAs. ${ }^{2,17}$ However, once an aneurysm has ruptured, the morphology and hemodynamics might change. This may reduce the accuracy of the results and limit the possible use of those parameters in clinical rupture assessments.9 Therefore, analyses of unruptured IAs with high rupture risk might provide more meaningful and reliable findings.

TABLE 1. Clinical, morphological, and hemodynamic characteristics of PCoA aneurysms

\begin{tabular}{llccc}
\hline \multicolumn{1}{c}{ Variable $^{*}$} & ONP, $\mathrm{n}=14$ & Rup, $\mathrm{n}=33$ & Unrup, $\mathrm{n}=21$ & $\mathrm{p}$ Value \\
\hline Baseline characteristics & & & & \\
\hline Age, yrs & $62(58-76)$ & $58(52-64)$ & $63(57-69)$ & 0.094 \\
\hline Male & $3(21.4)$ & $7(21.2)$ & $4(19.0)$ & 0.978 \\
\hline Hypertension & $8(57.1)$ & $13(39.4)$ & $12(57.1)$ & 0.343 \\
\hline Diabetes mellitus & $3(21.4)$ & $2(6.1)$ & $5(23.8)$ & 0.129 \\
\hline Current smoker & $5(35.7)$ & $4(12.1)$ & $2(9.5)$ & 0.113 \\
\hline Morphological characteristics & & & $3.48(2.72-5.01)$ & $<0.0001$ \\
\hline Size, mm & $6.96(5.31-8.20)$ & $5.10(4.12-6.39)$ & $0.84(0.68-0.97)$ & $<0.0001$ \\
\hline AR & $1.59(1.12-2.08)$ & $1.27(0.91-1.51)$ & $1.42(0.90-1.76)$ & 0.003 \\
\hline SR & $1.95(1.70-2.64)$ & $2.04(1.76-2.55)$ & $0.04(0.03-0.10)$ & $<0.0001$ \\
\hline UI & $0.17(0.10-0.25)$ & $0.11(0.07-0.17)$ & $0.13(0.08-0.17)$ & $<0.0001$ \\
\hline NSI & $0.24(0.23-0.29)$ & $0.22(0.15-0.26)$ & $0.09(0.05-0.11)$ & $<0.0001$ \\
\hline El & $0.20(0.16-0.24)$ & $0.17(0.13-0.21)$ & & 0.050 \\
\hline Hemodynamic characteristics & & & $0.01(0.00-0.02)$ & 0.001 \\
\hline OSI & $0.03(0.02-0.06)$ & $0.02(0.01-0.03)$ & $0.80(0.61-0.98)$ & $<0.0001$ \\
\hline Normalized WSS & $0.46(0.33-0.73)$ & $0.52(0.41-0.82)$ & $0.00(0.00-0.01)$ & $0.03(0.01-0.09)$ \\
\hline Percentage of LSA & $0.10(0.02-0.26)$ & $0.013)$ &
\end{tabular}

$\mathrm{ONP}=$ unruptured $\mathrm{PCOA}$ aneurysms with ONP; Rup = ruptured $\mathrm{PCOA}$ aneurysms; Unrup = asymptomatic unruptured PCoA aneurysms.

${ }^{*}$ Variables are expressed as the median (interquartile range) or number of patients $(\%)$. 


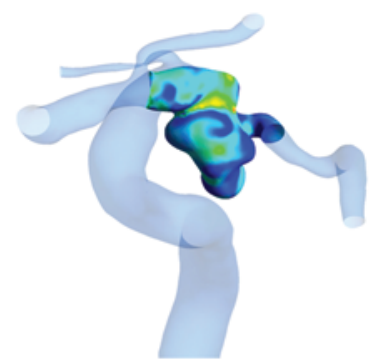

A

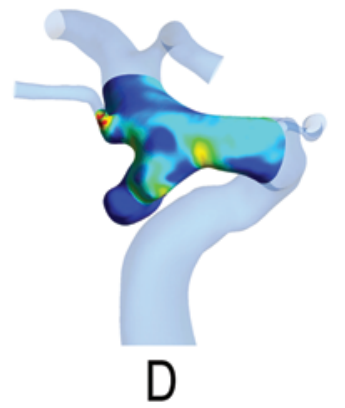

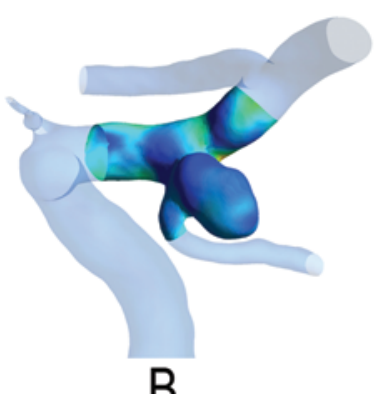

B

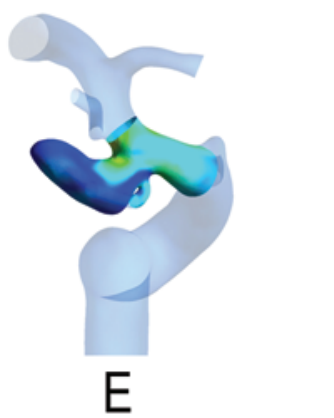

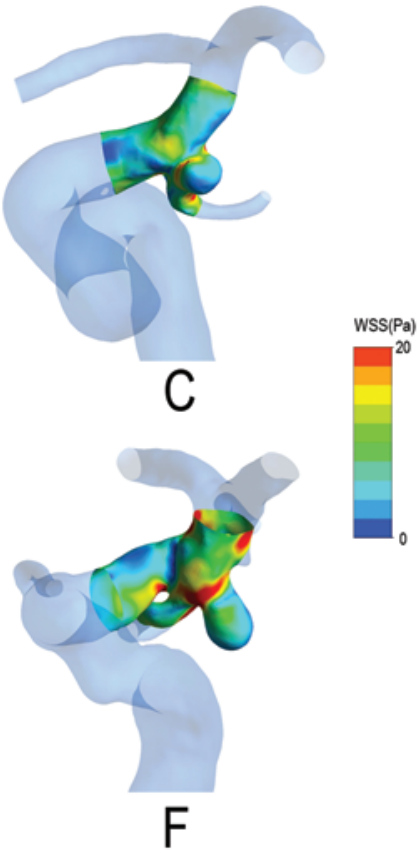

FIG. 1. Distribution of WSS. A and D: Unruptured PCoA aneurysms with ONP. B and E: Ruptured PCoA aneurysms. C and F: Asymptomatic unruptured PCoA aneurysms. Figure is available in color online only.

Several hemodynamic studies have reported some rare cases with radiographic records from not long before rupture. ${ }^{47,13}$ Duan et al. ${ }^{4}$ studied the hemodynamic and morphological features of several prone-to-rupture PCoA aneurysms and found that WSS might be a reliable discriminant of the rupture status of aneurysms. However, most of these studies were case reports or descriptive studies because it is hard to capture a specific status in clinical settings. Because ONP is a relatively common presenting sign of PCoA aneurysms, a considerable sample size can be acquired, which is important for accurate analyses.

The hemodynamic patterns of IAs correlate with their morphological features. Tremmel et al..$^{14}$ demonstrated that the luminal area of aneurysms with low WSS increased with an increasing SR. Boussel et al. ${ }^{1}$ found that aneurysm growth usually occurs in regions exposed to abnormally low WSS. In our study, we observed that all of the morphological parameters in the ONP group were significantly higher than those in the asymptomatic unruptured group, indicating that PCoA aneurysms with ONP are larger and more irregularly shaped. Accordingly, a significantly lower WSS was found in the ONP group compared with the asymptomatic unruptured group. Our results further suggested that a low WSS might be related to aneurysm enlargement and irregular shape.

Another advantage of the present study is that it focused only on PCoA aneurysms and did not combine all locations of aneurysms. A correlation between hemodynamics and aneurysm rupture has been widely demonstrated, but it is still controversial. Xiang et al. ${ }^{17}$ stated that a low WSS and a high OSI could be associated with aneurysm rupture, whereas Cebral et al..$^{2}$ argued that a high and concentrated WSS is related to rupture. We found that most CFD studies on rupture risk included aneurysms in multiple locations.
The conflicting findings of previous studies may be explained by the distinctive anatomical geometries and natural courses of aneurysms in different locations. ${ }^{11}$ Studies of a single location may be more reasonable for rupture risk assessment. Miura et al. ${ }^{11}$ demonstrated a correlation between low WSS and aneurysm-rupture status in an analysis of 106 middle cerebral artery aneurysms. Recent studies of the natural history of aneurysms have demonstrated that PCoA aneurysms are some of the most common aneurysms and have a relatively high rate of rupture..$^{12}$ However, few studies have comprehensively focused on only PCoA aneurysms and their clinical, morphological, and hemodynamic features. Therefore, we focused on PCoA aneurysms in this study.

There are several limitations to the present study. The small and imbalanced sample size of each group was the main limitation. The patient-specific models were simplified, and some arterial branches were artificially ignored. This study was a retrospective study, and although patientspecific models were applied in the CFD simulation, the boundary conditions were not patient specific. In addition, current CFD technology is time-consuming, which may limit its application in the timely decision making required in the treatment of unruptured aneurysms.

\section{Conclusions}

In this study, we compared the morphological and hemodynamic characteristics of unruptured PCoA aneurysms with ONP, ruptured PCoA aneurysms, and asymptomatic unruptured PCoA aneurysms. Unruptured PCoA aneurysms with ONP had a very different morphologicalhemodynamic pattern compared with asymptomatic unruptured PCoA aneurysms, and were similar to ruptured ones. The larger size, more irregular shape, and lower 
WSS might be related to the high rupture risk of PCoA aneurysms.

\section{Acknowledgments}

We gratefully acknowledge the Shanghai Supercomputer Center for its helpful provision of calculation software. We are also grateful for the useful suggestions of Dr. Jianping Xiang from the Toshiba Stroke Research Center, University at Buffalo, State University of New York. This study received funding from National Natural Science Foundation of China (No. 81171092), Shanghai Education Commission Innovation Fund (No. 14ZZ081), and Key Basic Scientific Project of Shanghai Science and Technology Committee (No. 11JC1415800).

\section{References}

1. Boussel L, Rayz V, McCulloch C, Martin A, Acevedo-Bolton $\mathrm{G}$, Lawton M, et al: Aneurysm growth occurs at region of low wall shear stress: patient-specific correlation of hemodynamics and growth in a longitudinal study. Stroke 39:29973002, 2008

2. Cebral JR, Mut F, Weir J, Putman C: Quantitative characterization of the hemodynamic environment in ruptured and unruptured brain aneurysms. AJNR Am J Neuroradiol 32:145-151, 2011

3. Dhar S, Tremmel M, Mocco J, Kim M, Yamamoto J, Siddiqui AH, et al: Morphology parameters for intracranial aneurysm rupture risk assessment. Neurosurgery 63:185-196, discussion 196-197, 2008

4. Duan G, Lv N, Yin J, Xu J, Hong B, Xu Y, et al: Morphological and hemodynamic analysis of posterior communicating artery aneurysms prone to rupture: a matched case-control study. J Neurointerv Surg [epub ahead of print], 2014

5. Golshani K, Ferrell A, Zomorodi A, Smith TP, Britz GW: A review of the management of posterior communicating artery aneurysms in the modern era. Surg Neurol Int 1:88, 2010

6. He X, Ku DN: Pulsatile flow in the human left coronary artery bifurcation: average conditions. J Biomech Eng 118:74-82, 1996

7. Hodis S, Uthamaraj S, Lanzino G, Kallmes DF, DragomirDaescu D: Computational fluid dynamics simulation of an anterior communicating artery ruptured during angiography. J Neurointerv Surg 6:e14, 2014

8. Jou LD, Lee DH, Morsi H, Mawad ME: Wall shear stress on ruptured and unruptured intracranial aneurysms at the internal carotid artery. AJNR Am J Neuroradiol 29:1761-1767, 2008

9. Kono K, Tomura N, Yoshimura R, Terada T: Changes in wall shear stress magnitude after aneurysm rupture. Acta Neurochir (Wien) 155:1559-1563, 2013

10. Lyrer P, Gratzl O: [Is early diagnosis possible in cerebral aneurysm? Symptoms of saccular cerebral aneurysms before the occurrence of massive subarachnoid hemorrhage. Literature review and discussion.] Schweiz Rundsch Med Prax 82:3-8, 1993 (Ger)
11. Miura Y, Ishida F, Umeda Y, Tanemura H, Suzuki H, Matsushima S, et al: Low wall shear stress is independently associated with the rupture status of middle cerebral artery aneurysms. Stroke 44:519-521, 2013

12. Morita A, Kirino T, Hashi K, Aoki N, Fukuhara S, Hashimoto $\mathrm{N}$, et al: The natural course of unruptured cerebral aneurysms in a Japanese cohort. N Engl J Med 366:2474-2482, 2012

13. Sforza DM, Putman CM, Scrivano E, Lylyk P, Cebral JR: Blood-flow characteristics in a terminal basilar tip aneurysm prior to its fatal rupture. AJNR Am J Neuroradiol 31:11271131,2010

14. Tremmel M, Dhar S, Levy EI, Mocco J, Meng H: Influence of intracranial aneurysm-to-parent vessel size ratio on hemodynamics and implication for rupture: results from a virtual experimental study. Neurosurgery 64:622-631, 2009

15. Valencia AA, Guzmán AM, Finol EA, Amon CH: Blood flow dynamics in saccular aneurysm models of the basilar artery. J Biomech Eng 128:516-526, 2006

16. Waga S, Otsubo K, Handa H: Warning signs in intracranial aneurysms. Surg Neurol 3:15-20, 1975

17. Xiang J, Natarajan SK, Tremmel M, Ma D, Mocco J, Hopkins LN, et al: Hemodynamic-morphologic discriminants for intracranial aneurysm rupture. Stroke 42:144-152, 2011

18. Xiang J, Tutino VM, Snyder KV, Meng H: CFD: Computational fluid dynamics or confounding factor dissemination? The role of hemodynamics in intracranial aneurysm rupture risk assessment. AJNR Am J Neuroradiol 35:1849-1857, 2014

19. Xu J, Yu Y, Wu X, Wu Y, Jiang C, Wang S, et al: Morphological and hemodynamic analysis of mirror posterior communicating artery aneurysms. PLoS One 8:e55413, 2013

20. Zanaty M, Chalouhi N, Tjoumakaris SI, Fernando Gonzalez L, Rosenwasser RH, Jabbour PM: Aneurysm geometry in predicting the risk of rupture. A review of the literature. Neurol Res 36:308-313, 2014

\section{Disclosures}

The authors report no conflict of interest concerning the materials or methods used in this study or the findings specified in this paper.

\section{Author Contributions}

Conception and design: Huang. Acquisition of data: $\mathrm{Yu}, \mathrm{Xu}$. Analysis and interpretation of data: Huang, Yu, Xu. Drafting the article: Lv. Critically revising the article: Lv. Reviewed submitted version of manuscript: Huang, Karmonik. Statistical analysis: Karmonik. Administrative/technical/material support: Liu. Study supervision: Liu.

\section{Correspondence}

Qinghai Huang, Department of Neurosurgery, Changhai Hospital, Changhai Rd., 168, Shanghai 200433, China. email: ocinhqh @163.com. 